\title{
Neue Vakzinierungsstrategien in der Immundermatologie und Allergologie
}

\author{
New Vaccination Regimens in Immunodermatology and Allergology
}

\section{Zusammenfassung}

In der klassischen Vakzineforschung wurde bisher versucht, eine möglichst effiziente Immunantwort gegen spezifische Antigene zu induzieren und gleichzeitig eine wirksame Gedächtnisimmunität zu erzeugen. Die Substanzen für diese Verwendungsart wurden vielfach erfolgreich in der Infektiologie sowie experimentellen Tumortherapie eingesetzt. In den letzten Jahren wurden jedoch zunehmend Vakzinierungsstrategien zur Modulation einer Immunantwort oder sogar zu deren Regulation und Suppression entwickelt. Gemeinsames Ziel dieser Forschungsarbeiten ist es, neue Behandlungsmöglichkeiten für bereits existierende, fehlgerichtete Immunantworten im Rahmen von Autoimmunerkrankungen zu entwickeln, die spezifisch, effektiv und nebenwirkungsarm sind. In diesem Zusammenhang spielen dendritische Antigen-präsentierende Zellen eine herausragende Rolle, da durch sie nicht nur T-Zellen Antigen-spezifisch aktiviert, sondern auch in ihrer Effektorfunktion moduliert und inhibiert werden können. Sowohl reife als auch unreife dendritische Zellen konnten in experimentellen Modellen für die Therapie von organspezifischer Autoimmunität erfolgreich eingesetzt werden. Es liegen sogar schon erste Ergebnisse über die Modulation einer anti-viralen Immunantwort im Menschen durch dendritische Zellen vor. In diesem Artikel wird der aktuelle Kenntnisstand über den Einsatz von dendritischen Zellen zur Induktion einer Immunregulation sowie Immuntoleranz dargestellt und diskutiert.

\section{Abstract}

Classical vaccination regimens play an important role against the spread of infectious agents or for the treatment of existing malignancies. The common goal of these vaccination efforts is to induce and maintain antigen-specific immunity including a robust memory response. However, in the recent years vaccination strategies have been developed, which aim to modulate and regulate immune responses. A growing body of evidence exists that different vaccination methods can even be used to suppress an ongoing immune response. These data built the rationale for the new development of immunosuppressive therapies for a safe, effective and well-tolerated treatment of organ-specific autoimmune diseases in dermatology and other clinical fields. Dendritic cells play a prominent role in this context since they not only activate but are also able to modulate and regulate the effector function of T cells. Dendritic cells have already been successfully used for the experimental treatment of autoimmune diseases in animal models. Dendritic cells can induce, stimulate and expand regulatory/suppressor T cells, which in turn actively inhibit effector T cells. Moreover, dendritic cells have been recently used in vivo to modulate an anti-viral immune response in humans. Therefore, dendritic cells appear to be promising tools for the development of new vaccination strategies which target the downregulation of (auto)immunity. In this article the latest experimental data on the use of dendritic cells for the induction of immunotolerance and the regulation of immunity are discussed in light of the recent literature. 
Einleitung

Das Gebiet der Immunologie wurde wesentlich durch die ersten Vakzinierungsuntersuchungen von Edward Jenner und Louis Pasteur beeinflusst. Seit diesen frühen klinischen Pionierleistungen wurde eine ständig wachsende Anzahl von Vakzinen entwickelt, die auch das Fach Dermatologie nicht unberührt gelassen haben. Durch Impfungen wurde die Inzidenz von Erkrankungen wie Masern, Röteln, Pocken aber auch Keuchhusten, Tetanus und Poliomyelitis drastisch gesenkt. Trotz des Anstiegs der vakzinierten Personenzahl von 5\% auf über 60\% bis Anfang 1990 , insbesondere in der so genannten Dritten Welt, versterben jedoch dort immer noch jährlich ca. 2 Millionen Kinder weltweit an Masern. Dies ist eine erschreckend hohe Zahl, die durch dementsprechende Impfungen absolut vermeidbar wäre. Daher ist es eines der gegenwärtigen Ziele der WHO, die Anzahl der Impfungen in den betroffenen Gebieten zu erhöhen.

Während die klassischen Vakzinierungsstrategien bisher darauf ausgerichtet waren, eine möglichst effiziente und effektive Immunantwort gegen bestimmte Haptene zu erzeugen, beschäftigen sich in jüngster Zeit immer mehr Wissenschaftler in der Vakzineforschung mit genau dem Gegenteil, nämlich der Unterdrückung einer Immunantwort durch Vakzinierung. Dies ist besonders auf dem Gebiet der Behandlung von Autoimmunerkrankungen von Bedeutung. Autoimmunität (z.B. Lupus erythematodes, Pemphigus vulgaris, bullöses Pemphigoid) ist gekennzeichnet durch das Auftreten von Autoantikörpern und/oder autoreaktiver T-Lymphozyten sowie der korrelierenden Organmanifestation der Erkrankung [10]. Eines der Ziele in der Etablierung dieser modernen Vakzinierungsstrategien ist es, die Produktion von Autoantikörpern zu unterdrücken bzw. autoreaktive T-Zellen zu inhibieren oder zu eliminieren. Um dies zu erreichen, ist die genaue Kenntnis einer Immunantwort, der Autoantigene sowie die Benutzung eines geeigneten Transportersystems derselben von Relevanz. Eine Vielzahl von Berichten sowie eigene Ergebnisse deuten darauf hin, dass aktivierte, reife dendritische Antigenpräsentierende Zellen (DC) in der Lagen sind, Immuntoleranz zu brechen und Autoimmunität zu induzieren $[9,19,20]$. Umgekehrt können unreife DC Immuntoleranz erzeugen und sogar mithelfen Immunantworten zu unterdrücken $[6,19]$. Im Folgenden ist der gegenwärtige experimentelle Kenntnisstand zum Einsatz von DC für die Behandlung von Autoimmunerkrankungen, Tumoren und Infektionen kurz zusammengefasst. Der Schwerpunkt dieses Artikels liegt auf der Entwicklung von DC für die therapeutische Regulation von Immunantworten.

\section{Regulation von Immunantworten durch DC}

DC befinden sich als unreife Antigen-präsentierende Zellen in einer Vielzahl von Geweben, u.a. der Epidermis als Langerhanszellen (Abb. 1a). Nach Aktivierung durch z.B. Infektionserreger oder andere Entzündungen wandern DC aus dem Gewebe in die regionalen Lymphknoten ein [1]. Dabei spielt die Stimulation von TOLL-Rezeptoren (z.B. TLR4) sowie TNF-Molekülen (z.B. CD40) auf der Zelloberfläche von DC eine wichtige Rolle. Innerhalb dieser Zeit durchlaufen DC einen mittlerweile gut charakterisierten Reifungsprozess, indem sie sich von einer primär Antigen-aufnehmenden in eine Antigen-präsentierende Zelle verwandeln, die in der Lage ist, T-Zellen Antigen-spezifisch zu stimulieren (Abb.1c). Eine wichtige Voraussetzung für diese effektive Aktivierung von T-Zellen ist die gemeinsame Expression des kognaten MHC-I/II-Antigen-Komplexes (Signal 1) sowie die zusätzliche Hochregulation von adhäsiven und kostimulatorischen Molekülen (Signal 2) auf der Zelloberfläche. Weiterhin produzieren reife DC Chemokine, die weitere T-Zellen anlocken können. Aus infektiös-entzündlichem Gewebe auswandernde aktivierte DC präsentieren jedoch nicht nur mikrobielle Antigene, sondern auch potenzielle Autoantigene, da sie nicht zwischen selbst und fremd unterscheiden können. Somit birgt jede Entzündung oder Infektion die Gefahr der Entwicklung bzw. Aktivierung von autoreaktiven T-Zellen. In der Tat wurde in der Maus nach Induktion einer Gastritis eine erhöhte Anzahl von gegen das mukosale Autoantigen ATPase gerichtete Effektor-T-Zellen nachgewiesen [21]. Das Immunsystem verfügt allerdings über verschiedene Schutzmechanismen Autoimmunität zu verhindern. $\mathrm{Zu}$ diesen gehört die Entwicklung von Toleranz sowie Anergie, die Depletion von autoreaktiven T-Zellen bzw. deren aktive Suppression durch suppressive T-Zellen. Interessanterweise wurde eine Beteiligung von DC an jeder dieser Immunmechanismen nachgewiesen, so dass sich DC nicht nur für die Induktion einer Immunantwort eignen, sondern auch potenzielle Kandidaten für deren Regulation darstellen $[6,19]$. Zur Zeit wird bereits experimentell versucht, diese regulatorischen Eigenschaften von DC im Hinblick auf ihre therapeutische Anwendbarkeit zu überprüfen.

In den letzten Jahren zeigten mehrere Berichte, dass gewebsständige DC an der Homöostase des entsprechenden Organs wesentlich beteiligt sind. Gewebsständige DC sind unreif aber nicht inaktiv (Abb.1b). Ortsständige DC der Darmmukosa sind an dem Abtransport von abgestorbenen Darmepithelzellen beteiligt, da Epithelzellüberreste in den intrazellulären Vesikeln von DC, die sich in den drainierenden Lymphknoten des Darmgewebes befanden, nachweisbar sind [19]. Für die Langerhanszellen der Haut wird eine ähnliche Zirkulation postuliert. Ebenso wurden DC in den Lymphknoten des Pankreas gefunden, welche Zellantigene der Langerhansschen Inseln, sie produzieren Insulin und werden beim juvenilen Diabetes mellitus durch autoreaktive T-Zellen zerstört, gebunden an MHC-Komplexe auf der Zelloberfläche trugen. Durch diese natürliche Zirkulation von ortsständigen DC werden regelmäßig die entsprechenden Gewebeantigene in den regionalen Lymphknoten T-Zellen präsentiert. Es kommt aber nicht zu einer T-Zellaktivierung, da das Antigen nur im Zusammenhang mit Signal 1 (MHC-Komplex), aber nicht zusammen mit Signal 2 (Adhäsions- und Kostimulationsmolekülen) von T-Zellen gesehen wird. Interagieren DC und T-Zellen lediglich über Signal 1, so führt diese Zell-Zellkommunikation zur Entwicklung von Toleranz bzw. Depletion der T-Zelle(n). Es wird vermutet, dass dies ein wichtiger peripherer Toleranzmechanismus ist, der die nicht perfekte, durch den Thymus gesteuerte, zentrale Toleranz ergänzt $[19,20]$.

\section{Bindung von löslichen Antigenen an unreife DC} zur Erzeugung von Immuntoleranz

Unreife DC sind auf die Aufnahme von Fragmenten (Phagozytose) sowie Flüssigkeit (Pinozytose) spezialisiert. Über beide Mechanismen können DC Bestandteile aus ihrer näheren Umgebung 

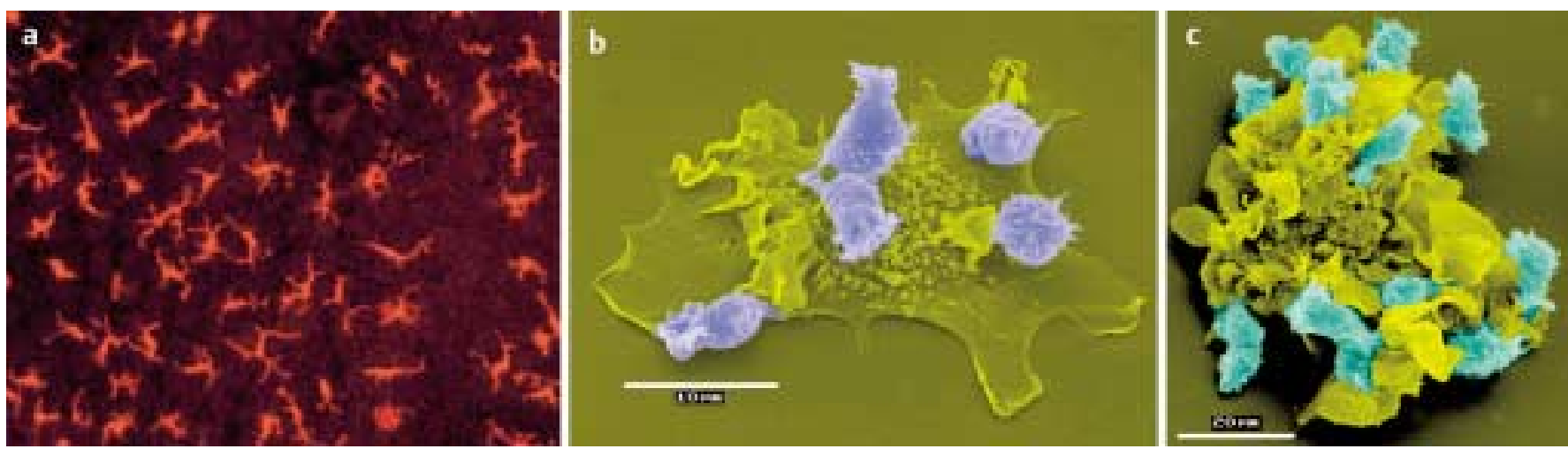

Abb. 1 Formen dendritischer Zellen. a Das Netzwerk von Langerhanszellen in der Mausepidermis. b Interaktion von einer unreifen humanen dendritischen Zelle mit CD4 ${ }^{+}$T-Zellen. $c$ Interaktion von einer reifen humanen dendritischen Zelle mit CD8 ${ }^{+} \mathrm{T}$-Zellen. internalisieren. Es wurden u.a. zwei Rezeptoren detektiert, die an diesen Aufnahmemechanismen beteiligt sind. Es handelt sich dabei um den Makrophagen-Mannose-Rezeptor (CD206) sowie DEC-205 (CD205). Besonders CD205 scheint für die weiteren Untersuchungen sehr interessant, da über diesen Rezeptor im Gegensatz zu über CD206 aufgenommene Antigene 30-100fach effizienter präsentiert wurden $[7,8,19]$. Zusätzlich ist dieser Rezeptor sowohl in der Maus als auch auf humanen DC, nicht aber auf T-Zellen und Makrophagen, nachweisbar. Weiterhin lässt sich CD205 in vivo auf DC in lymphatischem Gewebe darstellen. Ein natürlicher Ligand für CD205 ist bisher nicht identifiziert worden. Interessanterweise werden an CD205 gebundene und aufgenommene Antigene sowohl über MHC-Klasse I und auch MHC-Klasse II (Signal 1) präsentiert. Somit scheint der CD205-Rezeptor ein sehr geeignetes System darzustellen, um Antigene gezielt an DC zu binden, so dass diese anschließend präsentiert werden können. Während der Ligation des CD205-Rezeptors entsteht keine Ausreifung von DC, d.h. Signal 2 wird nicht exprimiert.

Für die Untersuchung, ob eine gezielte Antigenbindung an den CD205-Rezeptor experimentell durchführbar ist und ob infolge dessen autoreaktive T-Zellen deletiert werden können, wurde ein transgenes Mausmodell verwendet. Um die Bindung eines Peptids an den CD205-Rezeptor zu gewährleisten, wurde das Lysozym-Protein des Huhns an modifizierte anti-CD205-Antikörper gekoppelt [3]. Die Injektion dieser Antikörper in Mäuse führte zur Bindung an den CD205-Rezeptor und damit zur Internalisierung des Hühner-Lysozym-Proteins in DC. Anschließend wurden transgene $\mathrm{CD} 4^{+}-\mathrm{T}-Z$ Zllen, die einen gegen dieses LysozymProtein gerichteten T-Zellrezeptor tragen, in Antikörper-vorbehandelten Tiere inokuliert. Nach der Interaktion dieser Lysozymreaktiven T-Zellen mit Lysozym-präsentierenden DC wurde eine Zellteilung der T-Zellen nachgewiesen. Jedoch im weiteren Verlauf der Immunantwort kam es zu einer kompletten Depletion dieser T-Zellen. In einem analogen Versuch wurde das gleiche Ergebnis für die Elimination von Antigen-spezifischen CD8 ${ }^{+}-\mathrm{T}-Z \mathrm{Zl}-$ len gefunden. Zusammenfassend zeigen diese Daten, dass sich im experimentellen Modell die gezielte Anbindung von potenziellen Autoantigenen an den CD205-Rezeptor für die Elimination von Antigen-spezifischen autoreaktiven T-Zellen zu eignen scheint.
Diese Resultate bilden derzeit die Rationale den CD205-Rezeptor gezielt für die Anbindung von humanen Autoantigenen an DC zu erforschen. Humane Autoimmunerkrankungen mit bekannten Autoantigenen sowie nachweisbaren autoreaktiven T-Zellen sind beispielsweise Pemphigus vulgaris (Desmoglein 3), Pemphigus foliaceus (Desmoglein 1), bullöses Pemhigoid (BP180, PB230). Aber auch Kontaktallergien bei bekannten Kontaktallergenen könnten somit möglicherweise Antigen-spezifisch behandelbar werden.

\section{Verwendung von unreifen, tolerogenen DC als Vakzine}

DC lassen sich aus verschiedenen Geweben isolieren. Die dafür verwendeten Präparationstechniken führen allerdings unweigerlich gleichzeitig zur Aktivierung von DC. Aus diesem Grund und da in der Regel nicht ausreichende Mengen von DC für Vakzinierungen isoliert werden können, wurde die Kultur von DC aus peripheren Monozyten bzw. aus dem Knochenmark der Maus entwickelt. Mithilfe dieser Techniken lassen sich DC in größerer Anzahl generieren. Da die Kulturbedingungen definiert sind, ist diese Prozedur für die Gewinnung von DC äußerst effektiv und reproduzierbar. Durch die gewählten Kulturbedingungen lässt sich der Reifungs- bzw. Aktivierungszustand von DC steuern.

So führt die Zugabe von Interleukin(IL)-10 zu den Kulturbedingungen zu einer Hemmung der Ausreifung von DC. Im Mausmodell wurden IL-10-behandelte DC, die mit Proteinen beladen wurden, bereits erfolgreich eingesetzt, um eine Inhibition der Aktivierung von kognaten T-Zellen zu erreichen [18]. Ebenso spielt IL-10 bei der Entwicklung von Antigen-spezifischer Toleranz in der Maus eine wichtige Rolle. Aber auch mit Tumor-Nekrose-Faktor(TNF)- $\alpha$ aktivierte DC waren in der Lage, im experimentellen, allergischen Enzephalomyelitis-(EAE) Modell der Maus die Krankheitssymptome drastisch zu mildern [11]. So bewirkte die Injektion von Autoantigen-beladenen, TNF- $\alpha$ aktivierten DC, eine deutliche Unterdrückung von autoreaktiven T-Zellen und dadurch zur Abnahme der EAE-Symptomatik. Da TNF- $\alpha$ aktivierte DC u.a. signifikante Konzentrationen von IL-10 freisetzen, könnten die therapeutischen Effekte dieser aktivierten DC möglicherweise durch IL-10 vermittelt worden sein. Zusammenfassend können durch die Wahl der Kulturbedingung DC mit regulatorischen Eigenschaften generiert werden. 
Diese in der Maus generierten erfolgversprechenden Untersuchungen haben im Folgenden dazu beigetragen in vitro kultivierte humane unreife DC zu verwenden, um eine Antigen-spezifische Immunantwort im Mensch zu unterdrücken. Gesunde Probanden, die sich auf natürlichem Wege gegen das HämophilusInfluenza-Virus sensibilisiert hatten, wurden mit autologen unreifen, Influenza-Matrix-Protein beladenen DC behandelt [19]. Bei der anschließenden In-vitro-Untersuchung der Stimulierbarkeit von probandeneigenen $\mathrm{CD}^{+}$-T-Zellen gegen das Influenzavirus zeigte sich, dass die Immunantwort in diesen Zellen nicht mehr induziert werden konnte. Gleichzeitig traten im Blut der Gesunden IL-10-produzierende T-Zellen mit immunsuppressiven Eigenschaften auf. Nach ein paar Monaten verschwanden diese suppressiven T-Zellen wieder und die CD8 ${ }^{+}$-T-Zellen der Probanden waren wieder durch das Influenza-Virus aktivierbar. In einem Kontrollversuch führte die Injektion von autologen reifen, Influenza-Matrix-Protein-beladenen DC in denselben Individuen an einem späteren Zeitpunkt zu einer Expansion von Influenza-Matrix-Protein-reaktiven CD8+-T-Zellen [19]. Diese Untersuchungen zeigen eindrucksvoll die Plastizität von DC sowie die dadurch resultierende Immunantwort. Diese vielversprechenden Ergebnisse veranschaulichen, dass die Generierung von regulatorischer/tolerogener DC und deren Einsatz für die humane Immuntherapie prinzipiell möglich ist („proof of principle“).

\section{Aktivierung von suppressiven T-Zellen durch DC}

Der wiederholte Kontakt von naiven T-Zellen mit unreifen DC führte in In-vitro-Studien zu der Entwicklung von regulatorischen T-Zellen mit immunsuppressiven Eigenschaften [4]. Auch bei der Injektion von unreifen DC in Probanden (s. o.) konnte das Auftreten von suppressiven T-Zellen beobachtet werden.

Regulatorische T-Zellen mit immunsuppressiven Eigenschaften wurden bereits vor mehreren Jahrzehnten erstmalig beschrieben [2]. Aufgrund der fehlenden Möglichkeiten sie zu isolieren und dadurch zu charakterisieren wurde ihre Existenz lange Zeit infrage gestellt. Erst durch die Entdeckung von $\mathrm{CD}^{+} \mathrm{CD} 25^{+}$-T-Zellen mit der Fähigkeit die Aktivierung von $\mathrm{CD}^{+} \mathrm{CD} 25^{-}-\mathrm{T}$-Zellen zu unterdrücken, war der Weg für weitere funktionelle Untersuchungen dieser Zellen geebnet $[5,12,14,17] . \mathrm{CD}^{+} \mathrm{CD} 25^{+}$suppressive T-Zellen $\left(\mathrm{T}_{\mathrm{s}}\right)$ können durch Zell-Zellkontakt die Stimulation und Proliferation von $\mathrm{CD}^{+} \mathrm{CD}_{25}$-T-Zellen aktiv inhibieren $[13,15] . T_{s}$ sind anerg, d.h. es findet keine Proliferation nach Stimulation über den T-Zellrezeptor statt. Diese Anergie lässt sich durch die Zugabe von IL-2 und/oder IL-15 in vitro aufheben [16]. Mittlerweile wurden von verschiedenen Forschergruppen die Existenz von $\mathrm{T}_{\mathrm{s}}$-Subtypen, z. B. regulatorische $\operatorname{Tr} 1 \mathrm{CD}^{+}{ }^{+}$-T-Zellen oder Th3-Zellen, postuliert $[15,19]$. Der immunsuppressive Wirkungsmechanismus dieser Tr1-Zellen soll durch die Sekretion von IL-10 in hohen Konzentrationen mediiert werden. Gemeinsam haben diese verschiedenen regulatorischen T-Zellsubtypen, 1. ihre suppressiven Eigenschaften und 2. ihren Zustand der Anergie. In verschiedenen Untersuchungsmodellen konnte gezeigt werden, dass die Aktivierung von $T_{s}$ Antigen-spezifisch erfolgen muss, jedoch die induzierte Immunsuppression dieser Zellen Antigen-unspezifisch erfolgt $[5,16]$. Weiterhin können $T_{s}$ die Entwicklung von Autoimmunität, zumindest im Mausmodell, unterdrücken [14]. Das Immunsystem von immundefizien- ten Mäusen kann durch den Transfer von $\mathrm{CD}^{+} \mathrm{CD} 25^{-}$und $\mathrm{CD} 4^{+}$ $\mathrm{CD}^{+} 5^{+}$teilweise rekonstituiert werden. Werden allerdings nur $\mathrm{CD}^{+}{ }^{+} \mathrm{CD} 25^{-}$ohne $\mathrm{CD}^{+} \mathrm{CD} 25^{+}$-T-Zellen transferiert, kommt es zur Entwicklung von Autoimmunität im Empfängertier. Die nachträgliche Applikation von $\mathrm{CD}^{+} \mathrm{CD} 25^{+}$unterdrückt den Autoimmunphänotyp [12]. Diese Transferexperimente zeigen die Wichtigkeit von $\mathrm{T}_{\mathrm{s}}$ im Rahmen der Kontrolle von Autoimmunität. Ob die Funktion von $\mathrm{T}_{\mathrm{s}}$ auch eine Rolle bei der Entwicklung von Autoimmunerkrankungen im Menschen spielt, ist derzeit Gegenstand intensiver Forschung. Insgesamt stellt die Induktion bzw. Aktivierung von $T_{s}$ eine hoffnungsvolle Alternative zum Einsatz von Medikamenten dar, das Immunsystem zu hemmen. Dabei wird zurzeit einerseits versucht, $T_{s}$ direkt über Zelloberflächenrezeptoren zu stimulieren, andererseits, die Induktion von $T_{s}$ indirekt über den Kontakt mit anderen immunkompetenten Zellen zu erreichen $[6,15,19]$. Da unreife DC $T_{s}$ induzieren können, bestände somit eine Möglichkeit der Induktion dieser Zellen [4]. Daher ist auch anzunehmen, dass ein Mechanismus von unreifen DC regulatorisch in Immunantworten eingreifen zu können möglicherweise über die Induktion von suppressiven T-Zellen mediiert wird, die wiederum die Stimulation von Lymphozyten hemmen können.

Vakzinierungsstrategien unter besonderer Berücksichtigung der Haut

In der Epidermis bilden Langerhanszellen ein Netzwerk von unreifen DC (Abb.1a). Nach Antigenaufnahme und Aktivierung wandern sie in die regionalen Lymphknoten ein, um T-Zellen Antigen-spezifisch zu stimulieren. Langerhanszellen eignen sich daher sehr gut als Zielzellen für Vakzine. In verschiedenen Untersuchungsmodellen wurden Gene, die für Tumorantigene codieren, aus Tumoren isoliert und mithilfe einer so genannten „gene gun" in die Haut von Mäusen inokuliert. In der Haut wurde die DNS von z.B. Keratinozyten und Langerhanszellen aufgenommen. Die Langerhanszellen wanderten in die Lymphknoten und es erfolgten eine Transkription sowie Translation des Tumorantigen-Gens, welches anschließend als Tumorantigen T-Zellen präsentiert wurde. Diese Tumorantigenpräsentation führte zur protektiven Immunisierung dieser Mäuse gegen den Tumor, so dass die Tiere eine anschließende Injektion des Tumors abgestoßen haben.

Eine weitere Möglichkeit Langerhanszellen für eine effektivere Antigenpräsentation auszunutzen, besteht in deren Voraktivierung. Eigene Untersuchungen an transgenen Mäusen, die IL-15 in der Haut überexprimieren zeigten, dass IL-15 Langerhanszellen voraktiviert und diese Stimulation zu einer effizienteren Sensibilisierung von Antigenen über die Haut führte (Loser K, Beissert S, unveröffentlichte Beobachtung). Dadurch ließen sich effizientere anti-virale Immunantworten generieren. Therapeutika, die diese Anforderungen möglicherweise erfüllen, stellt u. a. der topische Immunmodulator Imiquimod dar. Imiquimod wird zur Zeit für die Behandlung von Viruserkrankungen sowie kutanen Neoplasien eingesetzt. Es wäre durchaus vorstellbar, dass durch die entstehende Immunstimulation nach Applikation von Imiquimod Langerhanszellen aktiviert werden, so dass diese effektive Antigene mit dem Ziel präsentieren, eine verbesserte antivirale bzw. antitumorale Immunantwort zu erzeugen. Zusammen- 
fassend stellen Langerhanszellen ideale Zielzellen für die Vakzineforschung dar, da sie leicht zugängliche, manipulierbare, professionelle Antigen-präsentierende Zellen darstellen.

\section{Danksagung}

Diese Arbeit wurde durch die Deutsche Forschunggemeinschaft (DFG SFB 293 B8, DFG BE 1580/2-3, DFG BE 1580/6-1) unterstützt. Dr. Kurt Dittmar sowie Dr. Manfred Rohde, GBF, Braunschweig, stellten die elektronenmikroskopischen Bilder von humanen DC freundlicherweise zur Verfügung.

\section{Literatur}

${ }^{1}$ Banchereau J, Steinman RM. Dendritic cells and the control of immunity. Nature 1998; 392: 245-252

${ }^{2}$ Gershon RK, Kondo K. Cell interactions in the induction of tolerance: the role of thymic lymphocytes. Immunology 1970; 18: 723-735

${ }^{3}$ Hawiger D, Inaba K, Dorsett Y, Guo M, Mahnke K, Rivera M, Ravetch JV, Steinman RM, Nussenzweig MC. Dendritic cells induce peripheral T cell unresponsiveness under steady state conditions in vivo. J Exp Med 2001; 194: 769-779

${ }^{4}$ Jonuleit H, Schmitt E, Schuler G, Knop J, Enk AH. Induction of interleukin 10-producing, nonproliferating CD4(+) T cells with regulatory properties by repetitive stimulation with allogeneic immature human dendritic cells. J Exp Med 2000; 192: 1213 - 1222

${ }^{5}$ Jonuleit H, Schmitt E, Stassen M, Tuettenberg A, Knop J, Enk AH. Identification and functional characterization of human CD4(+)CD25(+) T cells with regulatory properties isolated from peripheral blood. J Exp Med 2001; 193: 1285-1294

${ }^{6}$ Jonuleit H, Schmitt E, Steinbrink K, Enk AH. Dendritic cells as a tool to induce anergic and regulatory $\mathrm{T}$ cells. Trends Immunol 2001; 22: $394-400$

${ }^{7}$ Mahnke K, Guo M, Lee S, Sepulveda H, Swain SL, Nussenzweig M, Steinman RM. The dendritic cell receptor for endocytosis, DEC-205, can recycle and enhance antigen presentation via major histocompatibility complex class II-positive lysosomal compartments. J Cell Biol 2000; 151: 673-684
${ }^{8}$ Mahnke K, Qian Y, Knop J, Enk AH. Induction of CD4+/CD25+ regulatory $\mathrm{T}$ cells by targeting of antigens to immature dendritic cells. Blood 2003; 101: 4862 - 4869

${ }^{9}$ Mehling A, Loser K, Varga G, Metze D, Luger TA, Schwarz T, Grabbe S, Beissert $\mathrm{S}$. Overexpression of CD40 ligand in murine epidermis results in chronic skin inflammation and systemic autoimmunity. J Exp Med 2001; 194: 615-628

${ }^{10}$ Mehling A, Beissert S. Dendritic cells under investigation in autoimmune disease. Crit Rev Biochem Mol Biol 2003; 38: 1-21

${ }^{11}$ Menges M, Rossner S, Voigtlander C, Schindler H, Kukutsch NA, Bogdan C, Erb K, Schuler G, Lutz MB. Repetitive injections of dendritic cells matured with tumor necrosis factor alpha induce antigen-specific protection of mice from autoimmunity. J Exp Med 2002; 195: 15 - 21

12 Sakaguchi S, Sakaguchi N, Asano M, Itoh M, Toda M. Immunologic selftolerance maintained by activated $\mathrm{T}$ cells expressing IL-2 receptor alpha-chains (CD25). Breakdown of a single mechanism of self-tolerance causes various autoimmune diseases. J Immunol 1995; 155: $1151-1164$

${ }^{13}$ Sakaguchi S. Regulatory T cells: key controllers of immunologic selftolerance. Cell 2000; 101: 455-458

${ }^{14}$ Sakaguchi S. Animal models of autoimmunity and their relevance to human diseases. Curr Opin Immunol 2000; 12: 684-690

${ }^{15}$ Shevach EM. CD4+ CD25+ suppressor T cells: more questions than answers. Rev Immunol 2002; 2: 389-400

${ }^{16}$ Shevach EM. Certified professionals: CD4(+)CD25(+) suppressor T cells. J Exp Med 2001; 193: F41 - 46

17 Shevach EM. Suppressor T cells: Rebirth, function and homeostasis. Curr Biol 2000; 10: R572 - 575

${ }^{18}$ Steinbrink K, Graulich E, Kubsch S, Knop J, Enk AH. CD4(+) and CD8(+) anergic $T$ cells induced by interleukin-10-treated human dendritic cells display antigen-specific suppressor activity. Blood 2002; 99: $2468-2476$

${ }^{19}$ Steinman RM, Hawiger D, Nussenzweig MC. Tolerogenic dendritic cells. Annu Rev Immunol 2003; 21: 685-711

20 Steinman RM, Nussenzweig MC. Avoiding horror autotoxicus: the importance of dendritic cells in peripheral $\mathrm{T}$ cell tolerance. Proc Natl Acad Sci USA 2002; 99: $351-358$

${ }^{21}$ Suri-Payer E, Amar AZ, McHugh R, Natarajan K, Margulies DH, Shevach EM. Post-thymectomy autoimmune gastritis: fine specificity and pathogenicity of anti H/K ATPase-reactive T cells. Eur J Immunol 1999; 29: 669-677

\section{Preisverleihung}

\section{Deutscher Hautkrebspreises 2003}

Der Deutsche Hautkrebspreis wird jährlich von der Deutschen Hautkrebs-Stiftung unter Vorsitz von Prof. Dr. Roland Kaufmann (Universitätshautklinik Frankfurt am Main) verliehen. In diesem Jahr erfolgte die Preisvergabe anlässlich der 26. Jahrestagung der Vereinigung für Operative und Onkologische Dermatologie (VOD) in Verbindung mit der 24. Jahrestagung der International Society for Dermatologic Surgery (ISDS) am 20. September 2003 in Berlin. Die Überreichung des Preises wurde dieses Mal persönlich durch die Schirmherrin der Deutschen Hautkrebsstiftung, Frau Petra Roth, vorgenommen, die sich in besonderem Maße für die Bekämpfung von Hautkrebs engagiert.

Bereits zum 3. Mal konnten mit dem in Höhe von $10000 €$ dotierten Deutschen Hautkrebspreis außerordentliche Leistungen auf klinisch-onkologischem bzw. experimentellem Gebiet ausgezeichnet werden. Die diesjährigen Preisträger, Frau Dr. med. Beatrice Schuler-Thurner von der Universitätshautklinik Erlangen und Herr PD Dr. med. Axel Hauschild vom Universitätshautklinikum Kiel erhielten die Auszeichnung zu gleichen Teilen. Frau Dr. Schuler-Thurner erhielt die Ehrung für hervorragende Arbeiten im Bereich der experimentellen Dermatoonkologie, die sich insbesondere mit der Entwicklung und Optimierung von Immuntherapien des malignen Melanoms mittels peptid-beladener dendritischer Zellen beschäftigen. Herr PD Dr. Hauschild wurde mit seinen Arbeiten auf dem Gebiet innovativer Chemotherapieformen beim malignen Melanom gewürdigt. 